\title{
Crianças Enuréticas e Crianças Encaminhadas para Clínicas-Escola: Um Estudo Comparativo da Percepção de seus Pais
}

\section{Enuretical Children and Referred Children for University Mental Health Services: A Comparative Study of Their Parents' Perception}

\author{
Erika de Oliveira Lino dos Santos \& Edwiges Ferreira de Mattos Silvares* \\ Universidade de São Paulo, São Paulo, Brasil
}

\begin{abstract}
Resumo
Foi aplicada a Lista de Verificação Comportamental para Crianças/Adolescentes de 4-18 Anos (CBCL/4-18) nos pais de 66 crianças de seis a dez anos com enurese primária noturna (falta de controle da micção durante o sono, nunca adquirida anteriormente) atendidas pelo Projeto Enurese do Instituto de Psicologia da Universidade de São Paulo e de 235 crianças da mesma faixa etária encaminhadas para atendimento psicológico em cinco clínicas-escola brasileiras para uma comparação das percepções dos pais de ambos os grupos quanto ao perfil comportamental de seus filhos. De modo geral, as crianças enuréticas obtiveram melhores resultados que as encaminhadas quanto à competência social e a problemas escolares, de contato social e atenção. Os resultados parecem sugerir que estratégias diferenciadas de atendimento psicológico para as duas populações devem ser adotadas, focadas no problema da enurese primária no caso do primeiro grupo e nos problemas de desempenho escolar e social no segundo.

Palavras-chave: Clínicas-escola; enurese; CBCL.
\end{abstract}

\begin{abstract}
The Child Behavior Checklist (CBCL) was completed by the parents of 66 children age six to ten years old with nocturnal primary enuresis (i.e., children who wet the bed only at night and have never attained nocturnal urinary control), under treatment at the Enuresis Project of the Institute of Psychology of the Universidade de São Paulo, and by the parents of 235 children referred for psychological counseling services at five Brazilian mental health centers for a comparison of their perceptions regarding to their children's behavior profile. In most cases, enuretical children have obtained better results than the referred children regarding social competence as a whole and to academic, social contact and attention problems. These results seem to show that distinct psychological counseling strategies should be adopted for the two populations, focused on the primary enuresis problem in the first group and on the social and academic problems in the second group. Keywords: Mental health services; enuresis; CBCL.
\end{abstract}

Estudos realizados com a população que procura atendimento para crianças nas clinicas-escola brasileiras - na maioria dos casos, pais que buscam auxílio psicológico para os filhos - mostram que as razões principais para esta procura são a presença, em seus filhos, de comportamentos explícitos, como agressividade e problemas em seguir regras, e problemas no desempenho escolar (Bernardes-

\footnotetext{
*Endereço para correspondência: Av. Prof. Mello Moraes, 1721, Bloco F, sala 30, Cidade Universitária, São Paulo, SP, 05508-900.E-mail: eolsantos@yahoo.com.br; efdmsilv@usp.br Agradecimentos à FAPESP pelo auxilio à pesquisa; à PróReitoria de Pesquisa da USP pela bolsa concedida à primeira autora que permitiu a realização deste trabalho, à Prof. Dra. Sonia Beatriz Meyer pela co-orientação, à Tatiana Tung Gerencer, à Caroline Guisantes de Salvo e aos colegas do Laboratório de Terapia Comportamental da USP pelo auxílio na coleta e análise dos dados e ao GT da ANPEPP (Atendimento psicológico em clínicas-escola) - grupo que forneceu os dados das cinco clínicas-escola. Os resultados deste trabalho foram parcialmente apresentados no Xl Simpósio da Associação Nacional de Pesquisa e Pós-Graduação em Psicologia (ANPEPP) e no XII Simpósio Científico loternocional da Universidade de São Paulo (SICUSP), em 2004
}

da-Rosa, Garcia, Domingos \& Silvares, 2000; Marturano, Linhares, Loureiro \& Machado, 1997). Entretanto, há um alto índice de desistência nestes atendimentos (Silvares, 2000), e um dos motivos para isso pode ser o tipo de atendimento oferecido pelas clínicas-escola, o qual talvez seja insatisfatório para as demandas dos clientes.

Para um melhor atendimento a estas crianças e adolescentes, tornam-se necessários estudos sobre a caracterização desta população, bem como sobre as queixas mais freqüentes. Um dos tipos de distúrbios que atingem crianças e adolescentes, cuja alta incidência na população e prevalência em clínicas psicológicas tornam de interesse sua investigação seria a enurese primária noturna - isto é, quando a criança nunca conseguiu atingir o controle esfincteriano durante as horas de sono. Crianças enuréticas com idade a partir de seis anos recebem atendimento na clínica-escola da instituição de origem de uma das autoras, coordenado pela segunda.

Atualmente, a enurese primária noturna tem sido considerada um problema primário. Isto significa que não tem 
origem em problemas emocionais ou comportamentais sendo estes últimos conseqüência e não causa da enurese (Redsell \& Collier, 2001). Corroborando esta tese, há estudos mostrando que métodos de intervenção mais diretos, como o uso do alarme de urina e treinamento para o controle esfincteriano, mostraram-se mais efetivos para o tratamento da enurese noturna que a utilização da psicoterapia breve (Christophersen \& Mortweet, 2001). Além disso, estudos indicam melhoras comportamentais e emocionais em crianças cujo foco de tratamento incidem apenas sobre a enurese. Levando em consideração estes estudos e a teoria da enurese noturna como um problema primário, o atendimento oferecido às crianças enuréticas em nossa instituição visa primordialmente a aquisição do controle esfincteriano. Neste atendimento, a terapia é associada ao uso de um aparelho de alarme de urina, de eficiência empírica comprovada há mais de 50 anos (Silvares, 2002). O aparelho é ligado a um tapete que fica sob o lençol. Se a criança começa a urinar, o alarme dispara, dando-lhe tempo de reter a urina e ir ao banheiro. Com o tempo, a pessoa torna-se capaz de identificar a sensação de bexiga cheia antes de começar a urinar e o alarme disparar. Este adjunto terapêutico de tratamento tem colaborado para o sucesso do tratamento psicológico, ajudando várias crianças e adolescentes a conseguir o controle vesical (Silvares, 2002).

Com relação ao perfil comportamental da população enurética, há estudos que apontam maior prevalência de problemas de atenção e de relacionamentos sociais que na população em geral, que são mais graves quanto mais velha for a criança ou adolescente (Redsell \& Collier, 2001). Em um estudo com crianças chinesas de seis a 16 anos onde foi utilizado o CBCL (Child Behavior Checklist), (Liu, Sun, Ushiyama, Li \& Okawa, 2000) verificou-se que, quanto mais tarde uma criança tivesse obtido o controle da urina, mais chances de ter problemas internalizantes, como ansiedade e depressão e de contato social, atenção, pensamento e seguir regras.

Uma revisão da literatura não evidenciou, até o presente momento, estudos semelhantes sobre a caracterização comportamental das crianças enuréticas brasileiras, tanto em relação à população em geral, quanto em relação à população encaminhada para algum tipo de atendimento psicológico. Este estudo pretende preencher esta lacuna, pois a avaliação dos recursos e problemas comportamentais da população com enurese primária noturna é fundamental para uma intervenção bem-sucedida.

O objetivo do presente estudo foi comparar as percepções de dois grupos de pais quanto à competência social e problemas de comportamento de seus filhos. No primeiro grupo estão os pais que procuraram algum tipo de atendimento psicológico para problemas diversificados de seu filho em cinco clínicas-escola do país; no segundo grupo, aqueles cujos filhos freqüentam ou buscaram atendimento psicológico para a solução de um problema específico: a enurese. Tal comparação permitiu uma caracterização comparativa, o que possibilita avaliar que tipos de atendimento psicológico, entre os já existentes, podem trazer mais benefícios para cada um desses grupos.
Nosso estudo pretendeu verificar em que medida as características dos dois grupos enumeradas na literatura também aparecem em nossa amostra avaliada pelo mesmo instrumento, com o objetivo de traçar diretrizes de atendimento que atendam às necessidades destes dois grupos: o de crianças cujos pais apontam queixas diversificadas e o de crianças cujos pais apontam enurese primária noturna.

\section{Método}

\section{Participantes}

Participaram do estudo 301 pais de crianças atendidas em cinco clínicas-escola brasileiras das seguintes cidades: São Paulo (SP), Ribeirão Preto (SP), Rio de Janeiro (RJ), Goiânia (GO) e Curitiba (PR). Destes, 235 tinham filhos atendidos em função de queixas diversificadas e $66 \mathrm{com}$ queixa de enurese.

\section{Instrumento}

A avaliação da percepção dos pais foi realizada por meio da aplicação da Lista de Verificação Comportamental para Crianças/Adolescentes de 4-18 Anos (CBCL), instrumento de avaliação psicológica criado no final da década de 70, por Achenbach, nos Estados Unidos. O instrumento tem como objetivo medir o grau de competência social e os problemas compor tamentais de uma criança ou adolescente de acordo com a percepção de seus pais ou cuidadores (Achenbach, 1991). O CBCL tem tido grande penetração internacional, com traduções para mais de 30 idiomas (Achenbach, 1991), principalmente devido 'ao rigor metodológico com que foi elaborado, por seu valor em pesquisa e pela utilidade na prática clínica’. (Bordin, Mari \& Caeiro, 1995, p. 55)

O CBCL /4-18 é um questionário com 138 itens, entregue ou lido aos pais para que forneçam a apreciação global e comportamental de seus filhos. Do total de itens, 20 são destinados à avaliação da competência social da criança e 118 relativos à avaliação de seus problemas de comportamento. Assim, o questionário lista uma série de comportamentos, desejáveis e disruptivos, e apresenta para cada um deles, uma gradação de freqüência que vai de "não ocorre" até "muito freqüente".

A avaliação do CBCL fornece um perfil da criança a partir de 11 escalas individuais: três de competência social - Atividades, Sociabilidade e Escolaridade -, cuja soma dá origem à Escala Total de Competência Social (CS); e oito de problemas de comportamento - Retraimento, Ansiedade/Depressão, Queixas Somáticas, Problemas com o Contato Social, Atenção, Pensamento, Comportamento de Quebrar Regras e Comportamento Agressivo -, cuja soma dá origem à Escala Total de Problemas de Comportamento (PC).

Em cada uma destas somas de escalas a criança/adolescente pode ser classificada como normal, limítrofe ou clínica em relação à amostra normativa de Achenbach (1991). A Escala Total de Competência Social e a Escala Total de Problemas de Comportamento são as mais utilizadas para 
Santos, E.O.L., \& Silvares, E.F.M. (2006). Crianças Enuréticas e Crianças Encaminhadas para Clínicas-Escola: Um Estudo Comparativo da Percepção de seus Pais.

pesquisas e em uso clínico, juntamente com as Escalas de Internalização e Externalização, que são respectivamente as somas das três primeiras e das duas últimas escalas de problemas de comportamento.

A pontuação bruta obtida no CBCL em cada uma das escalas é convertida nos chamados Escores T, possuindo pontos de corte para as faixas clínica, limítrofe e não clínica (A Tabela 1 apresenta os pontos de corte de cada uma das escalas individuais e totais). O conjunto dos Escores T obtidos e da classificação destes em clínico, limítrofe ou não clínico, resulta no perfil da criança/adolescente avaliado. Para este estudo a faixa limítrofe foi agrupada à faixa clínica para que fosse minimizada a ocorrência de crianças classificadas como não clínicas pelo perfil do CBCL embora na realidade precisem de ajuda psicológica ("falsos negativos"), conforme a recomendação de Achenbach (1991) para pesquisas que utilizam o CBCL.

\section{Procedimento}

Todas as clínicas-escola envolvidas no estudo ficaram sob a coordenação da segunda autora, que também ficou responsável pelo treinamento dos pesquisadores participantes na aplicação do CBCL.

Os CBCLs de pais com queixas diversificadas sobre os filhos foram coletados nas cinco instituições e os de pais com queixa de enurese, apenas na clínica-escola de São Paulo.

Os pesquisadores envolvidos no estudo de crianças com queixas diversificadas aplicaram o CBCL em pais e responsáveis que estavam procurando por atendimento psicológico para crianças entre 6 e 10 anos de idade, em uma das clínicas-escola participantes do projeto e aceitaram participar da pesquisa, nos dias em que estas abriam inscrições para os atendimentos. Se havia mais de um informante disponível para uma criança no momento da aplicação, pedia-se que aquele que melhor conhecesse a criança respondesse ao questionário. As aplicações foram feitas em entrevista única em uma sala da clínica-escola, com duração aproximada de 30 minutos e incluindo a assinatura prévia de um termo de consentimento.

Após a aplicação, a avaliação dos dados foi realizada em um software especialmente desenvolvido para esse fim, e os perfis decorrentes foram enviados para as pesquisadoras de São Paulo para que fossem realizadas as análises estatísticas da amostra. Aqueles que não dispunham deste software enviaram seus dados para as pesquisadoras de São Paulo, para que estas os quantificassem e gerassem os perfis. Em nossa instituição a primeira autora foi responsável pela coleta de 39 questionários e pela elaboração e envio dos relatórios devolutivos sobre os resultados dos questionários que foram enviados aos pais e responsáveis.

Dentre os CBCLs preenchidos pelos pais de crianças e adolescentes com queixa de enurese, foram selecionados pela primeira autora aqueles que foram preenchidos pelos pais de crianças de seis a dez anos no momento em que o atendimento foi iniciado. No caso de crianças que tinham os dois CBCLs, foi escolhido o da mãe. Os dados foram tabulados e avaliados da mesma forma que os do primeiro grupo.

\section{Resultados}

As amostras advindas de cada uma das clínicas-escola foram reunidas em um só grupo, denominado Triagem, para ser comparado com o grupo das crianças enuréticas, atendido pela clínica-escola de nossa instituição e que foi denominado Enurese.

Foi coletado um total de 301 CBCLs, sendo 235 de pais com queixas diversificadas e 66 de pais com a queixa de enurese. A distribuição da população dos dois projetos quanto ao sexo, idade das crianças e respondente do CBCL encontra-se na Figura 1.

Grupo Triagem

Grupo Enurese

Sexo
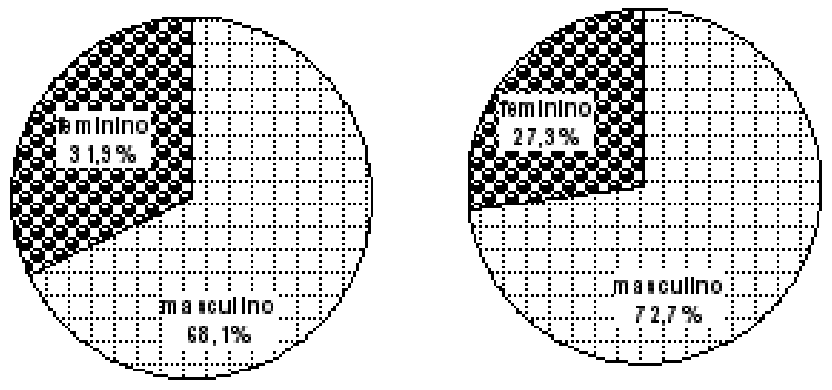

Idade
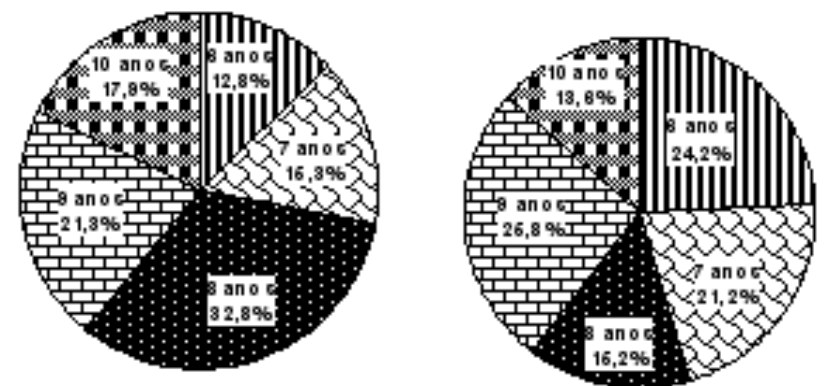

Respondente
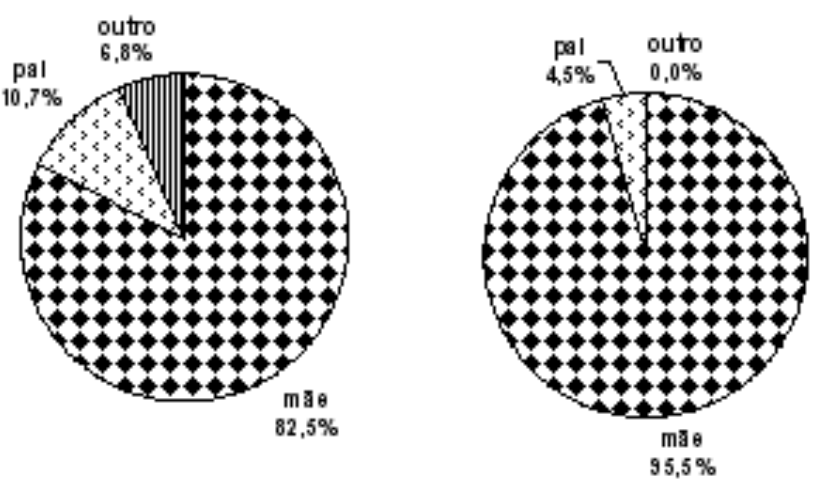

Figura 1. Distribuição por sexo, idade e respondente nos grupos triagem $(\mathbf{n}=235)$ e enurese $(\mathbf{n}=66)$. 
A análise das distribuições por sexo, idade e respondente nos dois grupos mostra haver poucas discrepâncias entre o Grupo Triagem e o Grupo Enurese: em ambos, apesar de haver distinção quanto à queixa, há uma predominância de crianças do sexo masculino, cujo CBCL foi respondido pela mãe. As análises de variância (ANOVA) entre os dois grupos mostraram que não há diferenças significativas entre eles quanto à distribuição destas três variáveis.

Foram calculadas as médias, em Escores T, que foram obtidas por cada grupo nas escalas de competência social e problemas de comportamento do CBCL (Tabela 1). A Escala de Competência Social Total só pode ser calculada quando são obtidos os escores das três escalas que a compõem (Atividades, Sociabilidade e Escolaridade), que por sua vez só podem ser calculadas caso todos os seus subitens estejam respondidos. Em nossa pesquisa as crianças que ainda não cursavam o Ensino Fundamental à época do preenchimento dos questionários não obtiveram o es- core da Escala de Competência Social Total porque a escala de Escolaridade não pôde ser calculada, pois os itens sobre desempenho em matérias escolares não puderam ser respondidos. Isso explica a variação de valores de $\underline{n}$ na Tabela 1 .

A grande maioria das médias das escalas situou-se fora da faixa clínica em ambos os grupos, com exceção das Escalas Totais de Competência Social e Problemas de Comportamento e da escala de Internalização nos dois grupos e da escala de Externalização no grupo Triagem. Este fato sugere que de uma forma global as crianças de ambos os grupos apresentam um déficit nas habilidades sociais e mais problemas de comportamento que a população geral. No grupo Triagem há, além disso, uma maior incidência de comportamentos problemáticos do tipo externalizante, que repercutem sobre o ambiente em que a criança está inserida.

Tabela 1

Escores T Médios dos Participantes dos Grupos Triagem e Enurese em cada Escala do CBCL

\begin{tabular}{|c|c|c|c|c|c|c|c|}
\hline \multirow{4}{*}{ escala } & \multicolumn{6}{|c|}{ grupo } & \multirow{4}{*}{$\begin{array}{c}\text { ponto } \\
\text { de } \\
\text { corte }^{*}\end{array}$} \\
\hline & \multirow{3}{*}{$\mathrm{n}$} & \multicolumn{2}{|c|}{ triagem } & \multicolumn{2}{|c|}{ enurese } & \multirow{3}{*}{ status } & \\
\hline & & escore & status & $\mathrm{n}$ & escore & & \\
\hline & & \multicolumn{2}{|l|}{$\mathrm{T}$ médio } & & T médio & & \\
\hline atividades & 234 & 35,55 & não clínico & 66 & 42,15 & não clínico & $\leq 33$ \\
\hline sociabilidade & 233 & 36,84 & não clínico & 66 & 40,68 & não clínico & $\leq 33$ \\
\hline escolaridade & 211 & 38,67 & não clínico & 60 & 43,62 & não clínico & $\leq 33$ \\
\hline escala total CS & 208 & 32,18 & clínico & 60 & 39,67 & clínico & $\leq 40$ \\
\hline retraimento & 235 & 62,37 & não clínico & 66 & 59,21 & não clínico & $\geq 67$ \\
\hline queixas somáticas & 234 & 56,61 & não clínico & 66 & 56,95 & não clínico & $\geq 67$ \\
\hline ansiedade/depressão & 235 & 61,23 & não clínico & 66 & 61,29 & não clínico & $\geq 67$ \\
\hline contato social & 235 & 61,96 & não clínico & 66 & 58,45 & não clínico & $\geq 67$ \\
\hline pensamento & 235 & 59,53 & não clínico & 66 & 57,24 & não clínico & $\geq 67$ \\
\hline atenção & 235 & 65,69 & não clínico & 66 & 60,86 & não clínico & $\geq 67$ \\
\hline quebrar regras & 235 & 59,81 & não clínico & 66 & 58,70 & não clínico & $\geq 67$ \\
\hline comp. agressivo & 235 & 62,37 & não clínico & 66 & 60,83 & não clínico & $\geq 67$ \\
\hline internalização & 235 & 61,38 & clínico & 66 & 61,00 & clínico & $\geq 60$ \\
\hline externalização & 235 & 60,54 & clínico & 66 & 59,56 & não clínico & $\geq 60$ \\
\hline escala total PC & 235 & 62,83 & clínico & 66 & 62,80 & clínico & $\geq 60$ \\
\hline
\end{tabular}

Nota. ${ }^{*}$ - Faixa limítrofe + faixa clínica.

Uma análise de variância multivariada mostrou que de modo geral houve uma diferença significativa entre os escores $\mathrm{T}$ dos dois grupos (lambda de Wilks $\Lambda=0,712$; $\left.\mathrm{F}_{(15,251)}=6,776 ; p<0,005\right)$, em especial em todas as escalas de competência social e nas escalas de Retraimento, Contato Social e Atenção, nas quais as crianças do grupo Enurese obtiveram escores mais afastados da faixa clínica que as crianças do Grupo Triagem (ver os valores de F para cada escala na Tabela 2). Estes dados sugerem que as crianças do Grupo Enurese, na percepção de seus pais, possuem menos problemas de competência social que as crianças do Grupo Triagem. 
Santos, E.O.L., \& Silvares, E.F.M. (2006). Crianças Enuréticas e Crianças Encaminhadas para Clínicas-Escola: Um Estudo Comparativo da Percepção de seus Pais.

Tabela 2

Valores de F Obtidos para cada Escala do CBCL na Comparação entre os Dois Grupos $(N=301)$

\begin{tabular}{lll}
\hline escala & g.l. & $\mathrm{F}$ \\
\hline atividades & 1,298 & $13,393^{* * *}$ \\
sociabilidade & 1,297 & $12,357^{* * *}$ \\
escolaridade & 1,269 & $12,607^{* * *}$ \\
escala total cs & 1,266 & $48,802^{* * *}$ \\
retraimento & 1,299 & $5,530^{*}$ \\
queixas somáticas & 1,298 & 0,111 \\
ansiedade/depressão & 1,299 & 0,002 \\
contato social & 1,299 & $6,853^{* *}$ \\
pensamento & 1,299 & 0,275 \\
atenção & 1,299 & $10,794^{* *}$ \\
quebrar regras & 1,299 & 0,835 \\
comp. agressivo & 1,299 & 0,804 \\
internalização & 1,299 & 0,070 \\
externalização & 1,299 & 0,373 \\
escala total pc & 1,299 & 0,000 \\
\hline
\end{tabular}

Notas. ${ }^{*}-\mathrm{p}<0,05 ;^{* *}-\mathrm{p}<0,01 ;{ }^{* * *}-\mathrm{p}<0,001$.
Foi realizada uma regressão múltipla, de método stepwise, para verificar se havia preditores entre os escores das escalas de problemas de comportamento, dos escores da Escala de Competência Social Total nos dois grupos. Foram encontrados modelos significativos para ambos, sendo que a escala de Problemas de Atenção é uma preditora importante no Grupo Enurese e no Grupo Triagem e a escala de Problemas com o Contato Social, preditora do Grupo Triagem (ver valores na Tabela 3).

Tabela 3

Valores Encontrados nos Modelos de Regressão Múltipla para os Grupos Triagem $(n=235)$ e Enurese $(n=66)$

\begin{tabular}{|c|c|c|c|c|}
\hline \multirow{5}{*}{ modelo } & \multirow[b]{3}{*}{$\mathrm{F}$} & \multicolumn{3}{|c|}{ grupo } \\
\hline & & \multicolumn{2}{|c|}{ triagem } & enurese \\
\hline & & \multicolumn{2}{|c|}{$22,238^{* *}$} & $16,054^{*} * *$ \\
\hline & g.l. & \multicolumn{2}{|c|}{2,204} & 1,58 \\
\hline & $\mathrm{R}^{2}$ ajustado & \multicolumn{2}{|c|}{0,171} & 0,203 \\
\hline \multirow[t]{2}{*}{$\begin{array}{l}\text { valores das } \\
\text { variáveis } \\
\text { preditoras }\end{array}$} & $\begin{array}{l}\text { variável } \\
\text { preditora }\end{array}$ & $\begin{array}{l}1 \text { - escala de } \\
\text { problemas } \\
\text { de atenção }\end{array}$ & $\begin{array}{l}2 \text { - escala de } \\
\text { problemas com } \\
\text { o contato social }\end{array}$ & $\begin{array}{l}1 \text { - escala de } \\
\text { problemas } \\
\text { de atenção }\end{array}$ \\
\hline & beta & $-0,265^{* *}$ & $-\mathrm{O}, 2 \mathrm{O} 2^{*}$ & $-0,466^{* *}$ \\
\hline
\end{tabular}

Nota. ${ }^{*}-\mathrm{p}<0,05 ; * * *-\mathrm{p}<0,01$.

Um teste qui-quadrado 2 x 4 com a distribuição dos resultados na faixa clínica do CBCL para as crianças de cada grupo mostrou que esta difere de forma significativa $\left(x^{2}=28,463\right.$, gl $\left.=4, p<0,005\right)$ entre as crianças dos dois grupos (Figura 2), com uma maior concentração de crianças clínicas nas duas escalas (Competência Social e Problemas de Comportamento) no Grupo Triagem (49,36\%) e de crianças não clínicas no Grupo Enurese (24,24\%). Apesar de a maioria das crianças em ambos os grupos estar na faixa clínica, no Grupo Enurese a distribuição é mais equilibrada que no Grupo Triagem, onde quase metade das crianças está situada na faixa clínica nas duas escalas totais, o que sugere uma diferença significativa entre os dois grupos quanto ao grau de comprometimento psicológico.

\section{grupo triagem}

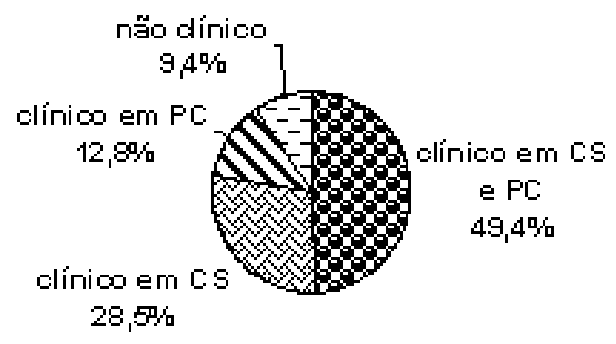

grupo enurese

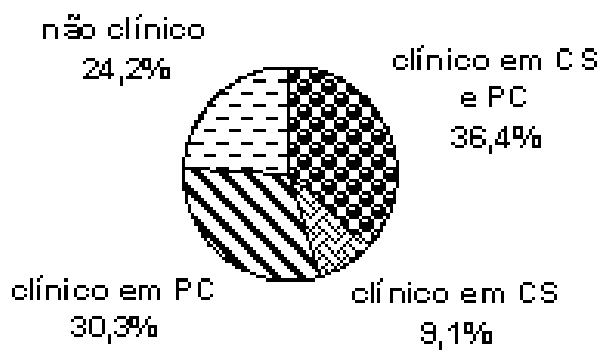

Figura 2. Distribuição dos resultados do CBCL dos grupos triagem $(\mathbf{n}=235)$ e enurese $(\mathbf{n}=66)$. 


\section{Discussão}

Em primeiro lugar é preciso ressaltar que os dados fornecidos pelo CBCL não nos falam sobre a criança, mas apenas sobre a percepção de seu pai ou mãe sobre ela. Uma avaliação completa sobre a criança deve integrar dados adquiridos através de entrevistas, observações e instrumentos de avaliação de mais de um ponto de vista, como os pais, professores, parentes e a própria (Achenbach, 1991). Assim, nossas conclusões sobre os resultados obtidos nas amostras dizem respeito tão-somente ao modo como os pais que participaram do presente estudo vêem seus filhos.

A comparação entre os escores médios obtidos pelas crianças do Grupo Triagem e do Grupo Enurese mostra que, de modo geral, as diferenças mais significativas entre os dois grupos estão nos escores de todas as escalas de competência social e nas escalas de Retraimento, Problemas com o Contato Social e Problemas de Atenção, sendo que as crianças do segundo grupo obtiveram escores mais altos nas escalas de competência social e mais baixos nas escalas de problemas de comportamento que o primeiro.

Estes dados sugerem que, de acordo com seus pais, as crianças enuréticas da amostra possuem mais habilidades e menos problemas em seus relacionamentos sociais e no desempenho escolar, o que reforça a teoria de que a enurese é um problema primário e que os problemas comumente associados a ela seriam conseqüências e não causas desta (Redsell \& Collier, 2001). Sabe-se também que a auto-estima e o ajuste emocional dessas crianças melhora consideravelmente após o tratamento da enurese (Azrin, Sneed \& Foxx, 1974; Redsell \& Collier, 2001). Pode-se concluir, portanto, que um atendimento psicológico mais focado em técnicas de controle da enurese, como é o oferecido em nossa instituição, pode ser mais rápido e benéfico para a criança ou adolescente, que um atendimento mais focado nas dificuldades emocionais e psicológicas coexistentes.

Os dados obtidos no Grupo Triagem corroboram os resultados do estudo de Bordin et al. (1995), onde as crianças encaminhadas para atendimento psicológico em geral apresentavam baixo rendimento escolar, maiores escores nos itens de Internalização e altos escores na escala de Problemas de Atenção na percepção de seus pais. É, em suma, uma população com dificuldades no desempenho acadêmico e que geralmente é encaminhada para atendimento por essa razão, e que, além disso, apresenta problemas de comportamento que ainda não se sabe ao certo se são causa ou conseqüência deste baixo desempenho (Marturano et al., 1997). O atendimento psicológico que é oferecido pelas clínicas-escola, portanto, deve levar em consideração estas especificidades de sua clientela ao traçar suas diretrizes.

Tais conclusões sobre os métodos de atendimento a estas crianças, entretanto, ainda não são definitivas: os resultados obtidos suscitam mais perguntas que respostas. Estas deverão ser respondidas em estudos futuros mais abrangentes, com amostras maiores e mais homogêneas, que permitam análises e comparações mais rigorosas.

\section{Referências}

Achenbach, T. M. (1991). Manual for the child behavior checklist/4-18 and 1991 profile. Burlington, VT: University of Vermont.

Azrin, N. H., Sneed, T. J., \& Foxx, R. M. (1974). Dry-bed training: Rapid elimination of childhood enuresis. Behavioral Research and Therapy, 12, 147-156.

Bernardes-da-Rosa, L. T., Garcia, R. M., Domingos, N. A. M., \& Silvares, E. F. M. (2000). Caracterização do atendimento psicológico prestado por um serviço de psicologia a crianças com dificuldades escolares. Revista de Estudos de Psicologia, 17(3), 5-14.

Bordin, I. A. S., Mari, J. J., \& Caeiro, M. F. (1995). Validação da versão brasileira do Child Behavior Checklist (CBCL). Inventário de comportamentos da infância e da adolescência: Dados preliminares. Revista $A B P-A P A L, 17(2)$, 55-66.

Christophersen, E. R., \& Mortweet, S. L. (2001). Treatments that work with children: Empirically supported strategies for managing childhood problems. Washington, DC: APA.

Liu, X., Sun, X., Ushiyama, M., Li, Y., \& Okawa, M. (2000). Attaining nocturnal urinary control, nocturnal enuresis, and behavioral problems in Chinese children aged 6 through 16 years. Journal of the Academy of Child and Adolescent Psychiatry, 39(12), 1557-1564.

Marturano, E. M., Linhares, M. B. M., Loureiro, S. R., \& Machado, V. L. S. (1997). Crianças referidas para entendimento psicológico em virtude de baixo rendimento escolar: Comparação com alunos não-referidos. Revista Interamericana de Psicologia, 31(2), 223-241.

Redsell, S. A., \& Collier, J. (2001). Bedwetting, behaviour and self-esteem: A review of the literature. Child, Care, Health and Development, 27(2), 149-162.

Silvares, E. F. M. (2000) Invertendo o caminho tradicional do atendimento psicológico em uma clínica-escola brasileira. Estudos de Psicologia, 5(1), 149-180.

Silvares, E. F. M. (2002). Família, enurese e intervenção clínica comportamental. In H. J. Guilhardi, M. B. B. P. Madi, P. P. Queiroz \& M. C. Scoz (Eds.), Sobre comportamento e cognição: Contribuições para a construção da teoria do conhecimento (Vol. 10, pp. 79-90). São Paulo, SP: ESETec.
${ }^{a}$ revisão: $01 / 08 / 2005$ Aceite final: 16/09/2005 